\title{
Overprescription of cholera vaccine to travellers by general practitioners
}

\author{
Alison Mott, Paul Kinnersley
}

\begin{abstract}
A questionnaire describing five hypothetical patients intending to travel to different countries was sent to 113 general practitioners, who were asked to state which patients they would recommend cholera vaccination to. The response rate was $80 \%$. The general practitioners' recommendations were compared with those of the Liverpool School of Tropical Medicine. Sixty three of 86 respondents recommended cholera vaccination when it would probably have been unnecessary. A review of common sources of information on cholera vaccination showed that general practitioners are given confusing or inappropriate advice.

General practitioners should be educated about when cholera vaccination is necessary; alternatively, the vaccine should be available only through special centres.
\end{abstract}

\section{Introduction}

Cholera epidemics occur only when there is overcrowding and poor sanitation, and endemic cholera rarely spreads to travellers. The incidence of cholera for each journey made by citizens of Europe or North America travelling abroad has been estimated to be one in $500000 .^{\prime}$ Among such travellers cholera is fairly benign with a low mortality. Cholera vaccine is unreliable, offering no more than $50 \%$ protection against the disease for only a few months. ${ }^{23}$ The low risk of contracting the disease and the unreliability of the vaccine has led to statements that cholera vaccination is not indicated for tourists visiting developing countries.

The World Health Organisation has stopped recommending cholera vaccination for travellers and has withdrawn its recognition of the international cholera vaccination certificate. ${ }^{+}$Others have recommended that the vaccine is given only to those "travelling extensively under arduous conditions in endemic areas" 2 and in countries where a certificate of vaccination is required.

Travel by British residents to countries outside Europe is increasing, ${ }^{6}$ and these travellers are at risk of considerable morbidity from preventable diseases ${ }^{7}$ and often ask their general practitioners for advice. The advice given varies widely and concentrates on the need for immunisation rather than information about drinking water or safe sexual practices. General practitioners seeking information to give to travellers may be confused as the information from medical newspapers and centres of excellence differs greatly. ${ }^{\circledR}$

Vaccination of travellers against cholera became common in the United Kingdom in the 1960s, and cholera vaccine is still widely administered by general practitioners. In 1987, 235000 prescriptions for it were written (prescribing office, Department of Health,

personal communication) at a cost of around $£ 1 \mathrm{~m}$. It continues to be described in review articles as a vaccine commonly given rather than one given in specific circumstances. " We designed this study to explore further the prescribing of cholera vaccine by general practitioners and to ascertain where general practitioners seek advice on this matter and what advice they receive.

\section{Subjects and methods}

We devised a questionnaire that described five hypothetical patients requesting advice before visits to different countries as follows: (1) a 32 year old man assessing an Oxfam health project in rural Sudan for one month, where there has been an outbreak of cholera; (2) a 25 year old man going on a sailing holiday along the south west coast of Turkey with occasional stops along the coast; (3) a 30 year old woman going on an exclusive two week holiday in Egypt, who had been reassured by her travel agent about the quality of the food and the accommodation; (4) a 22 year old student trekking in Nepal and returning through Kathmandu; (5) a 40 year old man on a five day business trip to Singapore.

The questionnaire was sent to 113 general practitioners chosen by selecting alternate doctors from the 225 principals on the 1988 list of the South Glamorgan Family Practitioner Committee. The general practitioners were asked to identify which travellers they would offer cholera vaccine to, according to their normal practice. They were free to refer to sources of information and were asked to identify those they used. A reminder was sent to those who had not responded three weeks after the initial mailing. The same questionnaire was sent to the Liverpool School of Tropical Medicine to establish an expert opinion. We also reviewed nine common sources of information on cholera vaccination that were available to general practitioners to see whether they would have recommended vaccination for the five patients in the questionnaire.

\section{Results}

We received 68 responses from the general practitioners initially and a further 22 after the reminder; this gave an overall response rate of $80 \%$. Four responses were not usable in the analysis owing to the manner in which the questionnaire had been completed.

All 86 general practitioners agreed that the traveller to Sudan should be offered vaccination. Twenty three stated that this was the only patient requiring vaccination. The expert source recommended vaccination only for this patient. The traveller to Nepal would have been advised to have vaccination by 61 respondents. Thirty five, 32 , and 28 respondents recommended vaccination for the travellers to Egypt, Turkey, and
General Practice,

Rusholme Health Cent

BrMed f 1990;300:25-6 
Recommendations for vaccination against cholera for travellers to five different countries according to nine common sources available to general practitioners

\begin{tabular}{lccccc}
\hline & \multicolumn{5}{c}{ Destination of traveller } \\
\cline { 2 - 5 } \multicolumn{1}{c}{ Source of information } & Sudan & Turkey & Egypt & Nepal & Singapore \\
\hline $\begin{array}{l}\text { Pulse foreign travel guide (1988 Dec 3) } \\
\text { Doctor magazine (1989 Feb 2) }\end{array}$ & Yes & No & No & No & No \\
Monthly Index of Medical Specialities (MIMS) & Yes & Yes & Yes & Yes & Yes \\
(Feb 1989) & Yes & No & No & Yes & No \\
DHSS leaflet Before You Go (SA40) (Feb 1988) & Yes & Yes & Yes & Yes & Yes \\
DHSS Immunisation against Infectious Diseases & Yes & No & No & No & No \\
Thomas Cook Group & Yes & No & No & No & No \\
Trailfinders (travel agent) & Yes & No & Yes & Yes & No \\
DHSS International Relations Division & Yes & Yes & No & Yes & No \\
Communicable Disease Surveillance Centre & Yes & No & No & No & No \\
\hline
\end{tabular}

Singapore respectively. Twenty five respondents recommended vaccination for all five travellers.

The 86 general practitioners each answered questions on five patients. If these patients are considered as a cohort of 430 patients 240 would have been recommended vaccination, whereas the Liverpool School of Tropical Medicine would have recommended vaccination for only 86 . This represents overprescribing of $280 \%$. As travellers to the Sudan are probably fewer than those to Nepal, Singapore, Egypt, or Turkey this is probably an underestimate.

Sixty five doctors said that they had used a source of information. These sources ranged from free weekly medical newspapers to specialist centres of tropical medicine. The commonest source was Pulse magazine. The sources produced a range of advice (table). Four recommended the vaccine only for the visitor to the Sudan; these four were either linked to specialists in infectious diseases or quoted specific specialist centres. Two sources recommended vaccination for all five travellers. Of the 62 general practitioners who quoted a source for their information, $30 \mathrm{did}$ not do as it had recommended; all 30 recommended vaccination to more patients than their source advised.

\section{Discussion}

The general practitioners provided information about hypothetical travellers, but our results probably indicate their behaviour with real patients. The results suggest that many general practitioners vaccinate travellers whom expert sources state do not need vaccination. The sources of information commonly used by general practitioners gave different recommendations for vaccination, some of them being inappropriate. Campbell drew attention to the use of unsolicited publications as sources of advice for travellers. ${ }^{10}$ Our results suggest a need for monitoring of sources of information. Some of the general practitioners used sources that made appropriate recommendations but they did not follow those recommendations. Thus the provision of up to date information is not necessarily enough to change behaviour.

There are many reasons why general practitioners may continue to recommend cholera vaccine. Some may believe that the disease is still a risk despite reference to up to date information. Others may fail to get clear or correct information. Patients may pressure doctors to prescribe, or the doctor may believe that patients will be vaccinated less safely abroad. The fear of litigation for negligence, or the patient's need for a cholera certificate, could also influence the doctor.

Though vaccination against cholera is safe in itself, there are risks to the patient from inappropriate vaccination. The injection may induce a sensitivity reaction or local symptoms at the injection site. Repeated vaccinations are likely to produce adverse reactions, and it should be avoided during pregnancy (manufacturer's recommendation, Wellcome). Perhaps most importantly, vaccination creates a false sense of security for both patient and doctor. The patient thinks he or she is protected yet will still be at risk from common gastrointestinal infections; the doctor considers that he or she has acted appropriately, particularly if so advised by a source of information.

The time taken to perform the vaccination will probably mean that vaccination replaces rather than accompanies advice. When patients consult a general practitioner before travelling abroad they want advice or action that will prevent them becoming ill. A discussion of the causes of travellers' diarrhoea will benefit them ${ }^{11}$; vaccination against cholera will not.

Financial costs both to the health service and to the patient should also be considered. Based on our estimate of overprescribing, $£ 0.65 \mathrm{~m}$ could be saved by general practitioners prescribing according to the guidelines of the Liverpool School of Tropical Medicine.

We believe that measures should be taken to educate general practitioners and perhaps share some of their burden of responsibility, otherwise a considerable number are likely to continue to vaccinate travellers to their disadvantage. There may also be a need to educate the public. An alternative would be to make cholera vaccine available only through special centres.

We thank the general practitioners who took part in this survey; Dr Malcolm Molyneux of the Liverpool School of Tropical Medicine for advice; and Drs Sarah Morgan and Keith Harding for their encouragement.

1 Morger $\mathrm{H}$, Steffen R, Schar M. Epidemiology of cholera in travellers, and conclusions for vaccination recommendations. Br.Med $\mathcal{f}$ 1983;286: 184-6. Bell DR. Protecting the travellers. In: Manson-Bahr PEC, Bell DR, eds Manson's tropical diseases. London: Baillière Tindall, 1987:1185-90.

3 Wolfe MS. Protection for travellers. In: Mandell G, Douglas R, Bennett J, eds Principles and practice of infectious diseases. New York: John Wiley, 1985:2307-16.

4 World Health Organisation. Vaccination certification requirements and healt advice for international travellers. Geneva: WHO, 1987.

5 DHSS. Immunisation against infectious diseases. London: HMSO, 1988.

6 Reid D, Cossar JH, Ako TI, Dewar RD. Do travel brochures give adequat advice on avoiding illness? Br Med f 1986:293:1472.

Cossar JH, Reid D, Grist NMR, et al. Illnesses assuciated with international travel: a ten year review. Travel Medicine International 1985;3:13-8.

8 Usherwood V, Usherwood TP. Survey of general practitioners' advice for travellers to Turkey: IR Coll Gen Pract 1989:39:148-9.

9 Peppiatt R. International travel medicine. $\mathcal{F} R$ Coll Gen Pract 1989;39:42-3.

10 Campbell $\mathrm{H}$. Imported malaria in the UK: advice given by general practitioners to British residents travelling to malaria endemic areas. $f R$ Coll ( $i e n$ ract 1987;37:70-2

11 Strum WB. Update on traveller's diarrhoea. Postgrad Med 1988;84:163-6.

(Accepted 27 October 1989)
The largeness of the issue of the BRITISH MEDICAL Journal (which it has again been necessary to increase materially since last year), together with the temporary disablement of some of the employés, caused an unavoidable delay in the postal delivery of some 5,000 copies of a recent number of the JOURNAL, which has led to many complaints. This general explanation will excuse individual replies. In view, however, of the increasing mechanical difficulties of passing through the press, cutting, and sewing, banding and stamping, so large an issue by the early post, it is now necessary to go to press at an early hour on Thursday. Our correspondents are therefore urgently requested to forward their communications as early as possible in the week when intended for the current issue; and to remember that Wednesday night's post is the latest by which even short and urgent communications can receive attention in that week.

(British Medical Fournal 1890;i:194) 